\title{
JETS OF FRAGMENTS FROM CATASTROPHIC BREAK-UP AND THEIR ASTROPHYSICAL IMPLICATIONS
}

\author{
G. MARTELLT, P. ROTHWELL, P.N. SMTTH, I. GIBLIN, J. MARTINSSON, E. DUCROCO \\ M. WETTSTETN \\ School of Mathematical and Physical Sciences, University of Sussex \\ Brighton BN1 90H Sussex \\ U.K. \\ M. DI MARTTNO \\ Osservatorio Astronomico di Torino \\ I-10025 Pino Torinese \\ Italy \\ P. FARINELLA \\ Dipartimento di Matematica, Universita' di Pisa \\ via Buonarroti, 2 \\ I-56127 Pisa \\ rtaly
}

ABSTRACT. We present some preliminary results of a series of catastrophic break-up experiments carried out in the open, against targets of natural and artificial rock, with and without a harder core. These experiments were aimed at investigating the outcomes of hypervelocity impact disruption phenomena, designed to understand the influence of large-scale collisions on the evolution of asteroids and other small solar system bodies. For the first time in this kind of experiments, evidence was found of collimated jets, i.e. the ejection of a statistically significant number of fragments all closely aligned about some preferential planes. Moreover, the presence of some groups of fragments lying close to each other on the ground was also detected.

\section{EXPERTMENTAL TECHNIQUE}

During the last few years the Space and Plasma Physics Group of the University of Sussex and the Planetology Group of the Turin Observatory have carried out a series of catastrophic fragmentation experiments in a travertine quarry (IMEG), located near Montemerano (Tuscany, Italy). The experiments were performed in the open, on a flat and relatively soft ground, in order to prevent secondary fragmentation of the primary fragments against the walls of a target chamber, and to record the uninterrupted ballistic trajectories of these fragments. We disrupted three targets, two of them being spheres of artificial rock (one with a core $10 \mathrm{~cm}$ in dianeter, harder than the surrounding "mantle") fabricated using alumina cement, carborundum $\left(\mathrm{SiC}_{2}\right)$ and water in the correct stechiometric ratio, the other consisting of an irregular parallelepipedal block of limestone veined by calcite crystals. The targets were placed on a steel table, provided in the centre with a circular hole having a diameter of $5 \mathrm{~cm}$, and were "impacted" from underneath (see Fig. 1). To simulate a high-velocity impact, we used a contact charge technique. This technique consists in filling a small cylindrical cavity in the target with plastic explosive and setting it off using a detonator. The momentum transferred by such an "equivalent projectile" is then measured using a ballistic pendulum. The pendulum consisted of a square block of steel weighing $4.7 \mathrm{~kg}$ suspended by 4 wires $6 \mathrm{~m}$ long. Upon detonation, the height gained by the pendulum was recorded by means of

\section{3}

A.C. Levasseur-Regourd and H. Hasegawa (eds.), Origin and Evolution of Interplanetary Dust, 383-386.

(c) 1991 Kluwer Academic Publishers, Printed in Japan. 

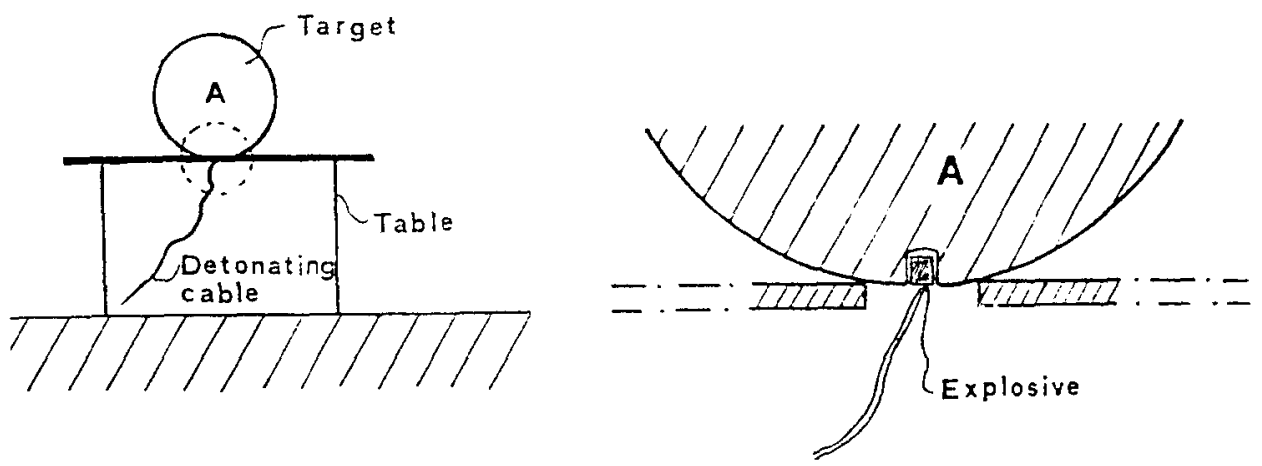

Figure 1. Experimental set-up.

fast-framing cameras. Using conservation of momentum, it was found that, within the errors, the equivalent mass of the "projectile", moving with the detonation velocity of the explosive $(6.1 \mathrm{~km} / \mathrm{s}$, see later $)$, was $1.80 \mathrm{~g}$. The explosive we used in the present series of experiments was Gelatine $2 \mathrm{~b}$, which has a detonation velocity of $6.1 \mathrm{~km} / \mathrm{s}$, density $1.42 \mathrm{~g} / \mathrm{cm}^{3}$ and specific energy $4.44 \mathrm{~kJ} / \mathrm{g}$. The value of the equivalent projectile energy over target mass (E/M) ratio was of the order of some units $\times 10^{7} \mathrm{erg} / \mathrm{g}$, which is well known to be the critical specific energy required to achieve catastrophic fragmentation of hard rock. We believe that the contact charge technique provides a fairly realistic simulation of a rock-rock impact, because the acoustic impedance of the target made of artificial rock is well matched by that of the exploding charge located against the target itself ( $7 \times 10^{5} \mathrm{~g} \mathrm{~cm}^{-2} \mathrm{~s}^{-1}$ ). In order to record the target disruption we used two CCD TV cameras operating at 50 fields per second and provided with a mechanical shutter giving an exposure time of $1 \mathrm{~ms}$. The CCD images have been analysed through a system which allowed two adjacent fields to be shown on a calibrated screen in rapid alternate motion, so that the displacement of the fragments from field to field could be measured accurately. Using a two-dimensional electronic cursor, it was also possible to plot the coordinates of any one fragment in a plane perpendicular to the axis of the cameras, and by simple software processing to obtain the component of the velocity of the fragments in this plane. The ground surrounding the table carrying the target was divided in 24 circular sectors of $15^{\circ}$ each by a graduated string lying on the ground, which allowed accurate polar coordinate measurements of the fragments lying on the ground up to a $30 \mathrm{~m}$ distance from the original position of the target. The fragments were numbered and stowed away in polythene bags for subsequent measurements.

\section{RESULTS}

An interesting feature, which has been clearly detectable because the experiments were performed in the open, is the formation of collimated jets, i.e., the presence of statistically significant numbers of fragments all very closely aligned about some radial directions. This effect has been found both with targets made of natural rock and with targets made of artificial rock (cored and uncored). The natural rock target consisted of limestone with the intrusion of thin calcite crystals in the form of rather regular planes. Upon reassembling the target, it was noted that these planes coincided with some of the fracture planes, and that some of the jets appeared to be ejected from these planes. Since well defined jets have been produced also from the target made of homogeneous artificial rock, it would appear that jets are associated with fracture planes, wherever they are formed. 
Figure 2 shows the frequency distribution of fragments as a function of the azimuthal angle for two targets, with and without the core. In order to enhance the presence of the jets and to show that a substantial fraction of the total mass can contribute to their formation, the numbers of fragments were multiplied by their respective mass. These plots exhibit peaks with high statistical significance. Since the experiments have been conducted in the open and in a relatively soft ground, it was also possible to observe some fragments grouped in "families" of up to some 10 members each. These fragments lied on the ground at small mutual distances (some centimeters) and many of their contours matched each other. Had the experiments been performed inside a target chamber, these fragments would have been scattered back at random into the chamber, and their common origin would most probably have been obscured.
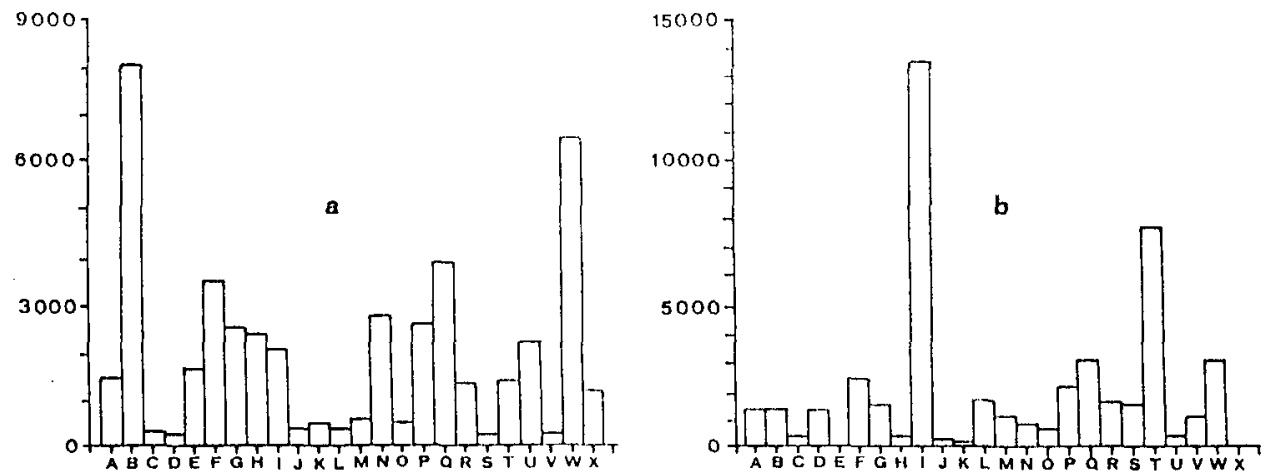

Figure 2. a) Fragment mass distribution from an artificial rock target (mass $=8.724 \mathrm{~kg}$, wi thout core) versus the azimuthal angle. In abscissae the capital letters indicate circular sectors of $15^{\circ}$ each, in ordinates the number of fragments multiplied by their mass in grams.

b) The same as in a) for an artificial rock target with core (mass $=8.632 \mathrm{~kg}$ ).

\section{DISCUSSION}

The existence of preferential planes and groupings among the ejected fragments is very interesting from the point of view of the formation of binary asteroids and of reaccumulated, self-gravitating asteroidal aggregates ("piles of rubble"), since these phenomena indicate that the fragment ejection velocity field after target break-up may be strongly non-isotropic. In other words, the relative velocity between two neighbouring fragments of mean radius $r$, ejected from a parent body of radius $R$, may be much less than the average value $\Delta \vee \underline{M}(r / R)$ Vmax, where $V \max$ is the peak ejection velocity. On the other hand, if a significant fraction of the mass of a target asteroid is dispersed "to infinity" against its self-gravity, this means that Vmax is at least $n 2 \mathrm{Ve}$, Ve being the target escape velocity. But the mutual escape velocity of two adjacent fragments is about $(\mathrm{Gm} / 2 \mathrm{r})^{1 / 2}$ (m being their mass),

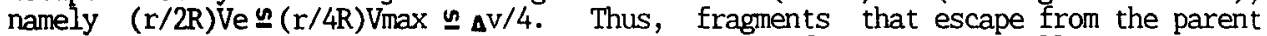
body can keep gravitationally bound to each other, forming eventually binary or reaccumulated bodies, only if some jetting is present in the initial velocity field. From the observational point of view, the recent discovery by radar observations of a binary asteroid having small size (of the order of $1 \mathrm{~km}$ ) and an Earth-approaching orbit (Ostro et al., 1990), suggests that the generation of gravitationally bound fragments during catastrophic collisions in the main asteroid belt may be fairly 
common. Further (indirect) evidence of the same process comes from the analysis of the spin rate distribution of small asteroids (Farinella et al., 1981; Binzel et al., 1989) and of the mass distribution of asteroid dynamical families (Zappala' et al., 1984; Chapman et al., 1989).

\section{ACKNOWLEDGMNTS}

This work was partially supported by NATO Grant $8605 / 43$.

\section{REFERENCES}

Binzel, R.P., Farinella, P., Zappala', V., and Cellino, A. (1989) Asteroids II University of Arizona Press, Tucson.

Chapman, C.R., Paolicchi, P., Zappala', V., Binzel, R.P., and Bell, J.F. (1989) Asteroids II, University of Arizona Press, Tucson.

Farinella, P., Paolicchi, P., and Zappala', V. (1981) 'Analysis of the spin rate distribution of asteroids', Astron. Astrophys. 104, 159-165.

Ostro, S., Chandler, J., Hine, A., Rosema, K., Shapiro, I., and Yeomans, D. (1990) 'Radar images of asteroid 1989 PB', Science 248, 1523-1528.

Zappala', V., Farinella, P., Knezevic, Z., and Paolicchi, P. (1984) 'Collisional origin of asteroid families: Mass and velocity distributions', Icarus $\underline{59}$, 261-285. 